\title{
A Single-center Comparison Using Exoskeleton Rehabilitation for Cerebrovascular Accidents and Traumatic Brain Injury in a Cohort of Hispanic Patients
}

\section{Lisa R Treviño}

Doctors Hospital at Renaissance

Kristina Vatcheva

University of Texas Rio Grande Valley

Michael E Auer

Doctors Hospital at Renaissance

Angela Morales

Doctors Hospital at Renaissance

Lama M Abdurrahman

University of Texas Rio Grande Valley

\section{Sarajova Viswamitra}

Doctors Hospital at Renaissance

Annelyn Torres-Reveron ( $\nabla$ anne.torres@dhr-rgv.com )

DHR Health Institute for Reseach and Development https://orcid.org/0000-0003-0347-5469

\section{Research}

Keywords: traumatic brain injury, robotics, cognition disorders, inpatient rehabilitation

Posted Date: July 17th, 2020

DOI: https://doi.org/10.21203/rs.3.rs-42275/v1

License: (c) (1) This work is licensed under a Creative Commons Attribution 4.0 International License. Read Full License 


\section{Abstract \\ Background}

Traumatic brain injury (TBI) is one of the leading causes of disability in the United States. The EKSO GT Bionics ${ }^{\circledR}(E K S O \AA)$ is a robotic exoskeleton approved by the Federal Drug Administration (FDA) for rehabilitation following a cerebrovascular accident (CVA or stroke) and recently received approval for use in patients with TBI. The aim of the study was to examine if the use of exoskeleton rehabilitation in patients with TBI will produce beneficial outcomes.

\section{Methods}

This retrospective chart-review reports the use of the $(\mathrm{EKSO} \otimes)$ robotic device in the rehabilitation of patients with TBI compared to patients with CVA. We utilized data from a single, private rehabilitation hospital for patients that received post-CVA or post-TBI robotic exoskeleton intervention. All patients that used the exoskeleton were discharged from the hospital between 01/01/2017 to 04/30/2020. Ninety-four percent of patients in the CVA groups and $100 \%$ of patients in the TBI group were of Hispanic or Latino ethnicity. Gains in total Functional Independence Measure (FIM), walking and cognition, and length of stay in the rehabilitation facility were measured.

\section{Results}

Patients in the TBI group $(n=11)$ were significantly younger than the patients in the CVA group $(n=66 ; p$ $<0.05$ ). Both groups spent a similar amount of time active, number of steps taken, and the number of sessions in the exoskeleton. Both groups also started with similar admission FIM scores. The FIM gain in the TBI group was similar to that of the CVA group (37.5 and 32.0 respectively). The length of stay between groups was not different either.

\section{Conclusions}

The use of exoskeleton rehabilitation in patients with TBI appear to produce similar outcomes as for patients with CVA, prompting further attention of this intervention for this type of injury. Trial registration: Retrospectively registered on 07/09/2020 in clinicaltrials.gov number NCT4465019.

\section{Introduction}

Traumatic brain injury (TBI) is one of the leading causes of disability in the United States. According to the Centers for Disease Control, approximately 3.2 to 5.3 million people are living with a TBI related disability in the United States ${ }^{1}$. Three specific age groups show a high risk of TBI: children aged 0-4, 
adolescents between 15-19 years of age, and adults 75 years and older ${ }^{2}$. The two leading causes of TBI are motor vehicle accidents and falls ${ }^{3,4}$. While the number of TBI related emergency department visits in the United States has increased by 54\% from 2006-2014, the mortality from TBI has decreased over the same time period ${ }^{4}$, suggestive of the positive impact of rehabilitation services. Severe to moderate TBI has the potential to create motor and ambulation deficits, which may resemble those presented in cerebrovascular accidents (CVA or stroke) ${ }^{5}$. Early intervention following TBI has been shown to produce improvements in post-TBI symptomathology ${ }^{6}$.

The beneficial use of robotic rehabilitation following a CVA has been long established in the field. The first studies that examined the acceptance of robotics in patients after CVA started in the nineties ${ }^{7}$. Consensus suggests that robotic-assisted gait training in patients after suffering a CVA or stroke is more likely to recover independent walking compared to those who did not use the robotic devices ${ }^{8,9}$. Studies also suggest that the use of robotics in rehabilitation could not only result in motor stills improvement but also transfer into other domains of daily living that require similar activities ${ }^{10}$, hence improving cognitive domains.

The use of robotics in rehabilitation medicine has become more popular due to the advantages it provides to the patients and the providers. The EKSO GT Bionics ${ }^{\circledR}$ (EKSO ${ }^{\circledR}$; Richmond, California, USA) is a robotic exoskeleton approved by the Federal Drug Administration (FDA) for rehabilitation following spinal cord injury, stroke, and recently received approval for acquired brain injury (including TBI) ${ }^{11}$. The use of this device provides an advantage to patients with these types of injuries by assisting them in walking with at least $3 / 5$ arm and upper muscle strength. It also provides a variable amount of power assistance to bilateral lower extremities and the ability for clinicians to control the device. EKSO ${ }^{\circledR}$ is approved for spinal cord injury up to $\mathrm{C} 7$ complete, any level of incomplete spinal cord injury, and hemiplegia due to stroke. The use of EKSO ${ }^{\circledR}$ in the rehabilitation of patients with TBI is starting to emerge due to the similarity of stroke-related impairments that can benefit from exoskeleton intervention. A recent case report of a young adult who suffered TBI, which resulted in right-side hemiplegia, revealed that the use of the EKSO ${ }^{\circledR}$ during a 4-week inpatient program significantly improved motor functional independence measure FIM score from admission to discharge ${ }^{12}$.

Not all patients are candidates for EKSO®-driven rehabilitation. All available bionic exoskeletons currently in the market have weight and height requirements as well as a set of contraindications for its use to ensure patient safety ${ }^{13}$. For example, bone mineral density is a metric for eligibility as to reduce the risk of fracture while using the device ${ }^{14}$. Moreover, the decision to employ bionic devices in a patient rehabilitation program is weighed by both provider recommendations and patient preferences. While some studies in patients with spinal cord injury and stroke demonstrate benefits from the use of exoskeletons, additional evidence for disorders that cause gait and mobility problems such as TBI are still necessary ${ }^{15,16}$. 
The main goal of our study was to examine the preliminary evidence of any beneficial outcomes, gathered at a single site-rehabilitation hospital using the EKSO ${ }^{8}$ in a cohort of patients who suffered TBI compared to patients who suffered a CVA. We compared our cohort of patients with TBI with all available data of patients with CVA who used the exoskeleton. Parameters used were the change (from admission to discharge) in total functional independence measure FIM, walking FIM gain, length of stay in the rehabilitation facility as well as cognitive FIM gain. Our central hypothesis was that the use of EKSO® improved function for TBI regardless of injury mechanism, similar to patients who suffered CVA and were also rehabilitated using the $\mathrm{EKSO}{ }^{\circledR}$. This hypothesis was based on the rationale that TBI and CVA produce related neurofunctional impairments ${ }^{17,18}$. To the best of our knowledge, this is the first study to analyze and report the use of the EKSO ${ }^{\circledR}$ in patients who suffered TBI. This study is also the first to report the outcomes of EKSO® use in a cohort of mostly Hispanic ethnicity patients.

\section{Methods}

\section{Study Design and Setting}

This is an observational, retrospective, chart review cohort study of patients from a private single rehabilitation hospital. Study approval was granted by the local Institutional Review Board and conformed to the Declaration of Helsinki and the US Federal Policy for the Protection of Human Subjects. Due to the retrospective nature of the study, a full waiver of authorization under the Health Insurance Portability and Accountability Act (1996) was submitted by the study team and approved by the Institutional Review Board.

\section{Participants}

\section{Inclusion and Exclusion criteria}

Subjects in the TBI group included patients who suffered a TBI and who used the EKSO $\circledast$ bionic exoskeleton during their rehabilitation treatment from 01/01/2017 to 04/30/2020. Subjects in the CVA group included all patients in the hospital that used the EKSO® during their rehabilitation process during the same period of the patients in the TBI group. Patients were excluded if their injury type was outside the scope of the current study. The registered nurse at the rehabilitation facility identified the patients based on the inclusion criteria, and this was verified by the doctor in physical therapy who oversees the rehabilitation hospital patient population. Approval and implementation of the EKSO® bionic device at the hospital commenced in 2014 for patients that suffered a CVA and spinal cord injury. Main indications for the use of the EKSO ${ }^{\circledR}$ during rehabilitation included a height range between 1.56 to 1.9 meters and a patient's weight equal to or less than $100 \mathrm{~kg}$. Stable blood pressure was required, with the ability to follow one to two steps commands, and the patient should also have demonstrated a normal range of motion in hips, knees, and ankles.

\section{Variables}


Demographic factors considered in this study were age at the time of admission into the rehabilitation facility (measured in years), and patient's sex (males or females). Ethnicity was self-reported and collected in the study, but it was not considered as a covariate since the vast majority of our cohort was of Hispanic origin. The overrepresentation of Hispanics/Latinos in the current cohort responds to the demographic distribution of the area the hospital serves. We collected information related to the impairment type produced by the CVA, and for the TBI, we collected the abbreviated injury scale (ASI) to determine the injury severity score (ISS).

The change in FIM score from admission to discharge was used as the primary outcome for this study. The FIM score evaluates the ability of the patient in day-to-day functions within three (3) major categories: self-care, mobility, and communication. To explore whether the use of EKSO $\circledast$ facilitates the improvement in mobility and communication, we also explored the motor FIM and social interaction FIM, problem-solving FIM, and memory FIM. In parallel to FIM, we evaluated how the length of stay in the facility affected the outcomes.

\section{Data sources/measurements}

Variables collected in this study were retrospectively obtained from the patient's electronic medical chart. All information presented in this study was part of the patient's standard of care. No additional intervention or data collection instrument was implemented. The standard of care of the patient is as follows: all potentially eligible candidates were identified and cleared by the physician with subsequent patient education regarding the use of the EKSO®. For the use of the exoskeleton during the rehabilitation process, signed informed consent was obtained from either the patient or designated family member, depending on the status of the patient. Data obtained during the active use of the exoskeleton was visualized in the screen of the EKSO ${ }^{\circledR}$ (time active, steps taken, etc.) and subsequently recorded in each patient's paper chart. This paper was then scanned and uploaded into the patient's electronic chart under his/her progress notes. The therapy technicians helped in the data recording process. All available data related to EKSO use for CVA and TBI at our facility were included in the current study. The primary goal of the data analysis was to gather preliminary comparative outcomes that could guide us into designing larger-controlled studies.

\section{Reduction of Bias}

To minimize selection bias in the current study, we used all patients that were clinically determined by the attending physician to meet the criteria for exoskeleton rehabilitation. Patients were included in the chart review regardless of the number of sessions of exoskeleton use. All therapist and nurses at our facility have been certified to use the FIM assessment tool and the data is entered using the Uniform Data System for Medical Rehabilitation (https://www.udsmr.org/) software integrated within the hospital medical record platform. The main data extractor in the study (A.M.) is the site expert for data management within the Uniform Data System. Quality of the data was verified by the lead doctor in Physical Therapy (M.E.A.). None of the patients that used exoskeleton in rehabilitation had missing data 
during the time period used for the current study and all patients in the cohort completed the recommended therapeutic program and were successfully discharged from the hospital.

\section{Exoskeleton use protocol}

The EKSO GT® was operated by a Licensed Physical Therapist who was certified to control the device. The device is only approved to be used in a clinical setting. For the purposes of this study, four different modes were utilized to engage and challenge the patients: Pre-Gait, First Step, Pro Step Plus, and 2 Free. Pre-Gait Mode focused on the following metrics to facilitate movement and active participation: bilateral weight shifting in standing with biofeedback, mini squats, and stationary unilateral lower extremity advancement. The First Step mode allowed the therapist to be in full control of all movement. The therapist triggered the initiation and execution of each step reciprocally with the push of a button. In the Pro Step Plus mode, there was an appropriate weight shift either done independently by the patient or facilitated by the therapeutic handling of the physical therapist. The patient was given the opportunity to initiate each step; the EKSO® device completed any incomplete steps. The most advanced mode is called 2 Free. This mode was used to challenge the patient across the continuum as they progress. The patient was responsible for initiating and executing each step. The therapist programmed resistance unilaterally or bilaterally to the lower extremities to increase the demand for the activity.

\section{Statistical methods}

Descriptive statistics for the entire study population were generated. Normally distributed continuous variables were described with means and standard deviations, medians and ranges were used for nonnormally distributed variables, and frequencies and column percentages summarized categorical variables. Differences in demographic and clinical characteristics between the two treatment groups were evaluated using Student t-test for independent samples for normally distributed continuous variables, Mann-Whitney U test for non-normally distributed continuous variables, and Fisher exact test for categorical variables. To test for the difference between the CVA and TBI groups in respect to sociodemographic and treatment measurements, paired t-test or Wilcoxon signed-rank test for continuous variables and Chi-square test/Fisher exact test for categorical variables were used. Shapiro-Wilk test for normality was conducted to determine the normality of the continuous variables' distributions. Linear regression analyses were carried out to evaluate the associations of each of the outcome variables with age, sex, and length of hospital stay, and with the injury groups (CVA and TBI) variable. All statistical tests were two-sided and were performed at a significance level of 0.05. All statistical analyses were conducted using SAS 9.4 (SAS Institute, Inc, Cary, North Carolina, USA.).

\section{Results}

\section{Participants}

From January 1st, 2017 to April 30th, 2020, rehabilitation was provided for 619 patients with CVA and 140 with TBI. A total of 66 (10.7\%) and 11 (7.9\%) patients met the criteria and agreed to use the 
exoskeleton for CVA and TBI respectively. All but one patient that used the exoskeleton during the retrospective review period were included in the study. This patient was excluded from the TBI group during the data quality review process since the exoskeleton was not used for gait therapeutic purpose, but solely as a tool to increase arousal in the individual.

\section{Outcome Analysis}

Patients who suffered TBI and used the exoskeleton for rehabilitation had a mean age of $45 \pm 25.81$, while patients who suffered CVA and used the exoskeleton had a mean age of $62.7 \pm 12.76$. Males were predominant $(77.9 \%)$ in the entire study population. Bivariate analyses showed that patients in the TBI group were significantly $(p<0.05)$ younger than patients in the CVA group (Table 1$)$. The mean number of exoskeleton training sessions was similar between groups with $3.5 \pm 1.86$ sessions in the TBI group and $3.5 \pm 2.93$ sessions in the CVA. Similarly, both groups spent equal amount of mobile minutes and took a similar amount of steps in the exoskeleton (Table 2). Average ISS for patients with TBI was $11 \pm 14.7$ points. In the CVA group, 33 patients had an impairment coded as 1.1 (stroke with left body involvement), 31 patients had an impairment code of 1.2 (stroke with right body involvement), and two patients were coded as 1.3 (stroke with bilateral involvement).

Further bivariate analyses revealed that none of the continuous variables measured were significantly different between groups (Table 2). On average, both groups had a similar number of exoskeleton rehabilitation sessions, minutes and steps taken during the sessions. The length of stay (LOS) and the length of stay efficiency (LSE) were also similar for both groups. The overall admission FIM rating was taken into consideration as a baseline measurement, showing a similar level of function between groups (Table 2). When comparing the change in overall FIM, the TBI group scored 5.5 points higher than the CVA group but there was no significant difference in this measurement. Similarly, the TBI group had a score that was 4.5 points over the CVA group for motor FIM change, but not significantly different. All three cognitive domains for FIM, memory, problem solving and social interaction scored similarly between groups during admission, discharge and overall change (Table 2).

Linear regression analyses demonstrated that there were no significant correlations of age with any of the FIM scores quantified or the length of stay.

\section{Discussion}

We present a comparative analysis amongst patients with a history of CVA and TBI, showing similar outcomes regardless of the injury type. Both CVA and TBI are non-progressive central nervous system condition that have very similar impairments ${ }^{19}$. Because of the similarity in impairment between CVA and $\mathrm{TBI}$, the use of exoskeleton rehabilitation for TBI patients was conceivable. While it is recognized that additional controlled clinical trials are needed for exoskeleton rehabilitation in $\mathrm{TBI}$, this preliminary study illustrates similar, positive outcomes in functionality to those patients treated with the exoskeleton which had a CVA. 
Patients within the TBI group were significantly younger than patients in the CVA group. There are several possible explanations for this observation. First, more than $80 \%$ of all stroke incidences across the nation occur in people 65 years and older ${ }^{20}$, in comparison to TBI for which two of the high risk groups are young children (age 0 to 4 ) and older adolescents (age 15 to $19 ;{ }^{1}$ ). Second, the use of the EKSO® required specific physical (e.g., height and weight) characteristics as well as physiological (e.g., bone density) to prevent injuries. It is well documented that older patients (> 50 years of age) are at higher risk of osteoporosis ${ }^{21}$, which is one of the main contraindications for the use of the exoskeleton, excluding them as potential users. Patient-preference is held at the highest priority when identifying eligible users. Anecdotal evidence from our hospital suggests that older patients are less likely to prefer rehabilitation with the exoskeleton, while younger patients are more eager to engage in the use of bionics and robotics, which could partially explain the age difference in our cohort of patients. Additional studies are needed to explore the generational gap in rehabilitation preferences, especially for the use of robotic devices.

Patients who suffer TBI, even when mild, could end up having impairments up to one year post injury ${ }^{22}$. Cognitive impairments after TBI could have even a longer sequelae, lasting up to 4 years, as previously reported ${ }^{23}$. The sequelae post-TBI that affects cognitive function is known as postconcussive syndrome ${ }^{24}$. To minimize its impact in patient's quality of life, rehabilitation approaches that promote neuroplastic changes in the brain, such as those produced by exoskeleton training, could result in an overall increase in functional domains for the patient after a TBI or CVA ${ }^{25}$. While the overall improvement in cognitive FIM scores in the current cohort of patients was small, a 1 point increase in FIM scores corresponds to an increase of 1.08 more likely to be discharged to the home rather than to institutionalized care ${ }^{26}$; hence decreasing healthcare cost on the long-term.

The use of robotic interventions in patients with TBI has lagged behind in comparison to the stroke field. A systematic review from 2011 reported ten randomized controlled trials for the use of robotic devices in CVA rehabilitation, but none for $\mathrm{TBI}^{27}$. However, recent reports indicate increased use of robotic rehabilitation following TBI; for example, to quantify the degree of impairment ${ }^{28}$, improve cognitive function ${ }^{29}$, to support treadmill training ${ }^{30}$, to measure brain activity while having robotic gait training ${ }^{31}$, and to increase gait velocity ${ }^{32}$. While the outcomes of these studies are mixed due to the differences in patient population, training techniques used, and type of equipment, it is promising that the field is recognizing how robotics present with a novel opportunity to improve quality of life in patients with TBI.

A recent study exploring the longitudinal effects of TBI revealed that higher total health burden was associated with poorer functional motor and cognitive trajectories ${ }^{33}$. To decrease the negative impact that TBI might have, it is hypothesized the continuous use of EKSO® as a rehabilitation device in our hospital will significantly continue to improve functional outcomes. Since the intervention with EKSO® revealed similar outcomes to the CVA patient cohort, even with small sample size, it is foreseen that many more patients eligible to use exoskeleton rehabilitation devices will greatly benefit from this therapeutic modality. 


\section{Study Limitations}

The literature on the use of exoskeleton devices for TBI resoundingly contains the limitation of sample size ${ }^{15}$, which restricts the power of the study to derive any reasonable outcomes. However, the limitation in the selection of a statistically significant sample size corresponds to the fact that patients need to meet a specific set of criteria to be eligible for rehabilitation with exoskeleton devices, as outlined in the methods section. It is also important to recognize that our study is based on a retrospective sample of patients, most of which were of Hispanic origin; thus, generalization to other ethnic groups might be limited. An additional consideration in this analysis was the fact that we did not control for comorbidities. While individual comorbidities may not directly affect outcomes observed in the use of exoskeleton, chronic conditions such as pressure sores, spinal instability, deep vein thrombosis, uncontrolled hypertension, among others ${ }^{13}$ are direct contraindications for the use of the device; hence limiting the pool of patients that can be included in the research studies. We did not control for severity of injury in the patients. However, our patients started with similar admission FIM scores, allowing us to have a similar baseline. Well-controlled randomized trials on exoskeleton rehabilitation are emerging as per published protocols ${ }^{34}$.

\section{Conclusions}

Physical therapists are more frequently employing robotic technology to enhance traditional rehabilitation therapy methods. The use of robotic technology to more specifically and deliberately treat patients with brain insults is allowing for increased intensity and duration of activity, and engagement of the patient with an activity of interest. This study demonstrated that gait training with the EKSO® GT Robotic Exoskeleton in patients with TBI as compared with a cohort of patients with CVA leads to similar improvements on FIM scores. Large multicenter randomized controlled trials comparing the use of the exoskeletons vs. traditional methods in the TBI population are warranted to verify and expand the preliminary findings of this study.

\section{Abbreviations}

CVA

cerebrovascular accident/stroke

TBI

traumatic brain injury

EKSO

EKSO GT Bionics ${ }^{\circledR}$ exoskeleton rehabilitation device

FIM

Functional Independence measure

AIS

Abbreviated Injury scale

ISS 
Injury severity score

\section{Declarations}

\section{Ethical Approval and Consent to Participate}

Study approval was granted by the DHR Health Institute for Research and Development Institutional Review Board and conformed to the Declaration of Helsinki and the US Federal Policy for the Protection of Human Subjects. Due to the retrospective nature of the study, a full waiver of authorization under the Health Insurance Portability and Accountability Act (1996) was submitted by the study team and approved by the Institutional Review Board.

\section{Consent for Publication}

All authors reviewed and approved the current version of the manuscript for publication.

\section{Availability of Supporting Data}

The datasets used and analyzed during the current study are available from the corresponding author on reasonable request.

\section{Competing Interests}

The following authors are employees of DHR Health System: LTR, MEA, AM, SV and ATR. Authors declare no other conflicts of interest and have no relationship of consulting employment or funding with the manufacturers of the devices used in the current study. None of the authors holds shares or stocks with EKSO Bionics Holdings.

\section{Funding}

Theresearch work presented received no funding by either the participating institutions or external sources.

\section{Authors Contributions}

LTR, MER and AM were responsible for the design of the study, data analysis and interpretation, and provided scientific content to the article. In addition, AM and MEAcollected the data. KV was responsible for data analysis and provided scientific and grammatical revisions to the manuscript. LMA was responsible for the scientific literature search and wrote the first draft of the Introduction. SV provided interpretation to the data and revision to the manuscript. ATR was responsible for the literature search, interpreted the statistical analyses, helped draft the manuscript results and discussion, and was responsible for the distribution and final approval by all authors. 
We appreciate the helpful review of an earlier version of this manuscript by Dr. Sohail Rao from DHR Health Institute for Research and Development.

\section{Author's information}

Authors e-mails in the same order of authorship: lis.trevino@dhr-rgv.com, kristina.vatcheva@utrgv.edu, m.auer@dhr-rgv.com, an.morales@dhr-rgv.com, lama.abdurrahman01@utrgv.edu,s.viswanitra@dhrrgv.com, anne.torres@dhr-rgv.com

\section{References}

1. Centers for Disease Control and Prevention. Report to Congress on Traumatic Brain Injury in the United States: Epidemiology and Rehabilitation. Atlanta, GA; 2015. doi:10.3171/2011.3.jns102010.

2. Faul M, Coronado V. Epidemiology of Traumatic Brain Injury. Vol 127.; 2015. doi:10.1016/B978-0444-52892-6.00001-5.

3. National Spinal Cord Injury Statistical Center. Facts and Figures at Glance. Birmingham, AL; 2019.

4. Centers for Disease Control and Prevention. Surveillance Report of Traumatic Brain Injury-Related Emergency Department Visits, Hospitalizations, and Deaths. United States; 2019. https://www.cdc.gov/traumaticbraininjury/get_the_facts.html.

5. Williams G, Lai D, Schache A, Morris ME. Classification of gait disorders following traumatic brain injury. J Head Trauma Rehabil. 2015;30(2):E13-23. doi:10.1097/HTR.0000000000000038.

6. Parrington L, Jehu DA, Fino PC, et al. The Sensor Technology and Rehabilitative Timing (START) Protocol: A Randomized Controlled Trial for the Rehabilitation of Mild Traumatic Brain Injury. Phys Ther. January 2020. doi:10.1093/ptj/pzaa007.

7. Dijkers MP, deBear PC, Erlandson RF, Kristy K, Geer DM, Nichols A. Patient and staff acceptance of robotic technology in occupational therapy: a pilot study. J Rehabil Res Dev. 1991;28(2):33-44. doi:10.1682/jrrd.1991.04.0033.

8. Mehrholz J, Thomas S, Werner C, Kugler J, Pohl M, Elsner B. Electromechanical-assisted training for walking after stroke. Cochrane database Syst Rev. 2017;5(5):CD006185. doi:10.1002/14651858.CD006185.pub4.

9. Bruni MF, Melegari C, De Cola MC, Bramanti A, Bramanti P, Calabrò RS. What does best evidence tell us about robotic gait rehabilitation in stroke patients: A systematic review and meta-analysis. J Clin Neurosci Off J Neurosurg Soc Australas. 2018;48:11-7. doi:10.1016/j.jocn.2017.10.048.

10. Fasoli SE, Adans-Dester CP. A Paradigm Shift: Rehabilitation Robotics, Cognitive Skills Training, and Function After Stroke. Front Neurol. 2019;10:1088. doi:10.3389/fneur.2019.01088.

11. Globe Newswire. Ekso Bionics ${ }^{\circledR}$ Receives FDA Clearance to Market its EksoNR ${ }^{\mathrm{TM}}$ Robotic Exoskeleton for Use with Acquired Brain Injury Patients.https://www.globenewswire.com/newsrelease/2020/06/25/2053400/0/en/Ekso-Bionics-Receives-FDA-Clearance-to-Market-its-EksoNRRobotic-Exoskeleton-for-Use-with-Acquired-Brain-Injury-Patients.html. Published June 25, 2020. 
12. $10.1109 /$ EMBC.2018.8512745

Nolan KJ, Karunakaran KK, Ehrenberg N, Kesten AG. Robotic Exoskeleton Gait Training for Inpatient Rehabilitation in a Young Adult with Traumatic Brain Injury. Conf Proc. Annu Int Conf IEEE Eng Med Biol Soc IEEE Eng Med Biol Soc Annu Conf. 2018;2018:2809-2812. doi:10.1109/EMBC.2018.8512745.

13. Palermo AE, Maher JL, Baunsgaard CB, Nash MS. Clinician-focused overview of bionic exoskeleton use after spinal cord injury. Top Spinal Cord Inj Rehabil. 2017;23(3):234-44. doi:10.1310/sci2303234.

14. Asselin PK, Avedissian M, Knezevic S, Kornfeld S, Spungen AM. Training Persons with Spinal Cord Injury to Ambulate Using a Powered Exoskeleton. J Vis Exp. 2016;(112). doi:10.3791/54071.

15. Dijkers MP, Akers KG, Dieffenbach S, Galen SS. Systematic Reviews of Clinical Benefits of Exoskeleton Use for Gait and Mobility in Neurologic Disorders: A Tertiary Study. Arch Phys Med Rehabil. 2019. doi:10.1016/j.apmr.2019.01.025.

16. Hesse S, Werner C. Connecting research to the needs of patients and clinicians. Brain Res Bull. 2009;78(1):26-34. doi:10.1016/j.brainresbull.2008.06.004.

17. Giles GM. Cognitive Versus Functional Approaches to Rehabilitation After Traumatic Brain Injury: Commentary on a Randomized Controlled Trial. Am J Ocupational Ther. 2010;64(May):182-5. doi:10.5014/ajot.64.1.182.

18. Capizzi A, Woo J, Verduzco-Gutierrez M. Traumatic Brain Injury: An Overview of Epidemiology, Pathophysiology, and Medical Management. Med Clin North Am. 2020;104(2):213-38. doi:10.1016/j.mcna.2019.11.001.

19. DiRocco PJ. Fitness Programming and Physical Disability. 1st ed. (Miller PD, editor). Champaing: Human Kinetics; 1995.

20. Blackwell DL, Villarroel MA. Summary Health Statistics for U.S. adults: National health interview survey. National Center for Health Statistics. . Published 2018.

21. Lane NE. Epidemiology, etiology, and diagnosis of osteoporosis. Am J Obstet Gynecol. 2006;194(2 Suppl):3-11. doi:10.1016/j.ajog.2005.08.047.

22. Nelson LD, Temkin NR, Dikmen S, et al. Recovery After Mild Traumatic Brain Injury in Patients Presenting to US Level I Trauma Centers: A Transforming Research and Clinical Knowledge in Traumatic Brain Injury (TRACK-TBI) Study. JAMA Neurol. 2019;76(9):1049-59.

doi:10.1001/jamaneurol.2019.1313.

23. Theadom A, McDonald S, Starkey N, et al. Social cognition four years after mild-TBI: An age-matched prospective longitudinal cohort study. Neuropsychology. 2019;33(4):560-7.

doi:10.1037/neu0000516.

24. Permenter CM, Fernández-de Thomas RJ, Sherman A. Postconcussive Syndrome. In: Treasure Island $(\mathrm{FL}) ; 2020$.

25. Berger A, Horst F, Müller S, Steinberg F, Doppelmayr M. Current State and Future Prospects of EEG and fNIRS in Robot-Assisted Gait Rehabilitation: A Brief Review. Front Hum Neurosci. 2019;13:172. 
doi:10.3389/fnhum.2019.00172.

26. Thorpe ER, Garrett KB, Smith AM, Reneker JC, Phillips RS. Outcome Measure Scores Predict Discharge Destination in Patients With Acute and Subacute Stroke: A Systematic Review and Series of Meta-analyses. J Neurol Phys Ther. 2018;42(1):2-11. doi:10.1097/NPT.0000000000000211.

27. Tomida K, Sonoda S, Hirano S, et al. Randomized Controlled Trial of Gait Training Using Gait Exercise Assist Robot (GEAR) in Stroke Patients with Hemiplegia. J stroke Cerebrovasc Dis Off J Natl Stroke Assoc. 2019;28(9):2421-8. doi:10.1016/j.jstrokecerebrovasdis.2019.06.030.

28. Logan LM, Semrau JA, Debert CT, Kenzie JM, Scott SH, Dukelow SP. Using Robotics to Quantify Impairments in Sensorimotor Ability, Visuospatial Attention, Working Memory, and Executive Function After Traumatic Brain Injury. J Head Trauma Rehabil. 2018;33(4):E61-73. doi:10.1097/HTR.0000000000000349.

29. Maggio MG, Torrisi M, Buda A, et al. Effects of robotic neurorehabilitation through lokomat plus virtual reality on cognitive function in patients with traumatic brain injury: A retrospective casecontrol study. Int J Neurosci. 2020;130(2):117-23. doi:10.1080/00207454.2019.1664519.

30. Esquenazi A, Lee S, Packel AT, Braitman L. A randomized comparative study of manually assisted versus robotic-assisted body weight supported treadmill training in persons with a traumatic brain injury. PM R. 2013;5(4):280-90. doi:10.1016/j.pmrj.2012.10.009.

31. Lapitskaya N, Nielsen JF, Fuglsang-Frederiksen A. Robotic gait training in patients with impaired consciousness due to severe traumatic brain injury. Brain Inj. 2011;25(11):1070-9. doi:10.3109/02699052.2011.607782.

32. Esquenazi A, Lee S, Wikoff A, Packel A, Toczylowski T, Feeley J. A Comparison of Locomotor Therapy Interventions: Partial-Body Weight-Supported Treadmill, Lokomat, and G-EO Training in People With Traumatic Brain Injury. PM R. 2017;9(9):839-46. doi:10.1016/j.pmrj.2016.12.010.

33. Kumar RG, Ketchum JM, Corrigan JD, Hammond FM, Sevigny M, Dams-O'Connor K. The Longitudinal Effects of Comorbid Health Burden on Functional Outcomes for Adults With Moderate to Severe Traumatic Brain Injury. J Head Trauma Rehabil. February 2020. doi:10.1097/HTR.0000000000000572.

34. Louie DR, Mortenson WB, Durocher M, Teasell R, Yao J, Eng JJ. Exoskeleton for post-stroke recovery of ambulation (ExStRA): Study protocol for a mixed-methods study investigating the efficacy and acceptance of an exoskeleton-based physical therapy program during stroke inpatient rehabilitation. BMC Neurol. 2020;20(1):1-9. doi:10.1186/s12883-020-1617-7.

\section{Tables}




\begin{tabular}{|lllll|}
\hline \multicolumn{4}{|l}{ Table 1: Demographic characteristics and exoskeleton use } & \\
\hline Variable & All subjects & CVA & TBI & P-value \\
\hline $\mathrm{n}(\%)$ & $77(100)$ & $66(86)$ & $11(14)$ & - \\
\hline Age in years, mean (SD) & $60.2(16.3)$ & $62.7(12.8)$ & $45(25.8)$ & 0.048 \\
\hline Hispanic/Latino, $\mathrm{n}(\%)$ & $73(95)$ & $62(94)$ & $11(100)$ & 0.402 \\
\hline Sex, $\mathrm{n}(\%)$ & & & & \\
\hline \multicolumn{1}{|l}{ Males } & $60(78)$ & $51(77.3)$ & $9(81.8)$ & 0.999 \\
\hline Females & $17(22)$ & $15(22.7)$ & $2(18.2)$ & \\
\hline EKSO sessions, mean (SD) & $3.3(2.8)$ & $3.3(2.9)$ & $3.5(1.9)$ & 0.767 \\
\hline EKSO minutes, mean (SD) & $32.2(35.25)$ & $31(35.4)$ & $39.4(34.9)$ & 0.467 \\
\hline EKSO steps, mean (SD) & $854.8(944.1)$ & $821.2(946.2)$ & $1056.6(950.49)$ & 0.448 \\
\hline LOS in days, mean (SD) & $20.9(8.77)$ & $21.1(9.2)$ & $19.5(5.5)$ & 0.588 \\
\hline LOS efficiency, mean (SD) & $1.8(0.9)$ & $1.8(0.9)$ & $2(0.7)$ & 0.414 \\
\hline
\end{tabular}

EKSO: EKSO GT robotic exoskeleton; LOS: length of stay; LOS efficiency refers to the points increase in overall functional independence measure (FIM) per day. 
Table 2: Functional independence measure scores (FIM) before and after exoskeleton use.

\begin{tabular}{|c|c|c|c|c|}
\hline Variable, mean (SD) & All subjects & CVA & TBI & $\begin{array}{l}\mathrm{P} \text { - } \\
\text { value }\end{array}$ \\
\hline Admission overall score & $41.9(16.3)$ & $42.3(16.4)$ & $39.5(16.5)$ & 0.598 \\
\hline Discharge overall score & $74.6(21.6)$ & $74.3(22.0)$ & $76.9(19.3)$ & 0.709 \\
\hline Change in overall score & $32.7(13.2)$ & $32.0(13.4)$ & $37.5(11.6)$ & 0.203 \\
\hline Change in motor score & $23.5(10.0)$ & $22.9(10.0)$ & $27.4(9.4)$ & 0.177 \\
\hline \multicolumn{5}{|l|}{ Cognitive domain scores } \\
\hline Change in social interaction & $1.4(1.0)$ & $1.4(1.0)$ & $1.3(0.9)$ & 0.683 \\
\hline Change in problem solving & $1.4(1.0)$ & $1.4(1.1)$ & $1.5(0.8)$ & 0.608 \\
\hline Change in memory & $1.5(1.0)$ & $1.5(1.0)$ & $1.6(0.7)$ & 0.673 \\
\hline
\end{tabular}

\section{Supplementary Files}

This is a list of supplementary files associated with this preprint. Click to download.

- RECORDstatementEKS007062020.pdf 